\title{
All-fiber zero-insertion-loss add-drop filter for wavelength-division multiplexing
}

\author{
Anthony S. Kewitsch, George A. Rakuljic, Phil A. Willems, and Amnon Yariv* \\ Arroyo Optics, Inc., Santa Monica, California 90404
}

Received June 18, 1997

\begin{abstract}
We developed and fabricated an all-fiber add-drop filter by recording a Bragg grating in the waist of an asymmetric mode converter-coupler formed by adiabatic tapering and fusing of two locally dissimilar, singlemode optical fibers. The insertion loss of the device was $\sim 0.1 \mathrm{~dB}$. A narrow spectral bandwidth $(<1 \mathrm{~nm})$ and a large add-drop efficiency $(>90 \%)$ were also demonstrated. In addition, the filter was polarization independent. (c) 1998 Optical Society of America

OCIS codes: $\quad 060.0060,060.4230,060.2310,060.2340,120.2440$.
\end{abstract}

Numerous optical filter concepts have been proposed for dense wavelength-division multiplexing (WDM) applications. ${ }^{1-5}$ For the lowest insertion loss and most reliably packaged device, an all-fiber solution is preferred. All-fiber add-drop filters have been demonstrated by combination of fiber Bragg gratings with a Mach-Zehnder interferometer ${ }^{6}$ or a polarization splitter, ${ }^{7}$ for example. A grating-assisted directional coupler $^{8}$ can also function as an add-drop filter, and an all-fiber device based on evanescent wave coupling and fiber Bragg gratings has been proposed. ${ }^{9}$

The present filter is based on grating-assisted mode conversion and backward coupling in the merged region of two dissimilar waveguides formed by adiabatic tapering and strong fusing of two single-mode optical fibers. From the theory of adiabatic following, ${ }^{10}$ the modes of the input fibers will evolve on a one-to-one basis into the first two eigenmodes of the merged region. These eigenmodes will propagate through the waist independently of each other, transform back into their original modes, and exit the device through their respective output fibers. This adiabatic mode evolution is lossless and free of optical cross talk.

Figure 1(a) illustrates the one-to-one relationship between input modes 1 and 2 in waveguides I and II, and the eigenmodes into which they are transformed. By recording a transversely asymmetric Bragg grating in the waist of the device, one can convert eigenmode 1 and backward couple it into eigenmode 2 , which will then exit the merged region as mode 2 via waveguide II, as shown in Fig. 1(b). This transformation will occur only at a specific wavelength $\lambda$ that satisfies the phase-matching or Bragg condition

$$
\beta_{1}(\lambda)+\beta_{2}(\lambda)=2 \pi / \Lambda_{g},
$$

where $\Lambda_{g}$ is the period of the grating and $\beta_{1,2}$ are the respective modal propagation constants. Because the eigenmodes and the grating fully overlap in the merged region, the coupling coefficient of this device is much larger than that of a grating-assisted directional coupler based on evanescent wave coupling.
If $\beta_{1}+\beta_{2} \neq 2 \pi / \Lambda_{g}$, input mode 1 will simply pass through the merged region, unaffected by the grating, and exit waveguide I unattenuated, as shown in Fig. 1(c). Conversely, if light that satisfies the Bragg condition is launched into waveguide II it will be added onto waveguide I by the grating. Thus this device functions as an efficient, narrow-bandwidth four-port all-fiber optical add-drop filter.

The structure of the filter that was fabricated is shown in Fig. 2. The diameter of the merged waist region was $7 \mu \mathrm{m}$. Inasmuch as identical starting fibers were used, we introduced the necessary waveguide dissimilarity by locally pretapering one of the two

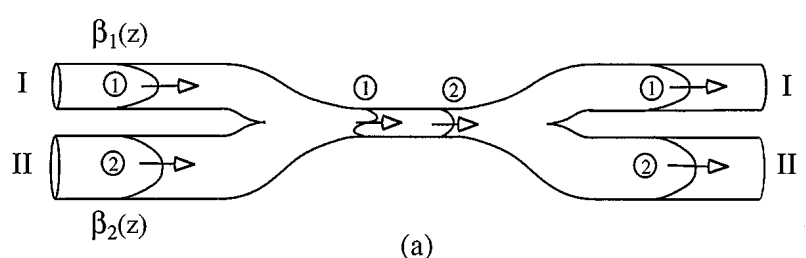

(a)

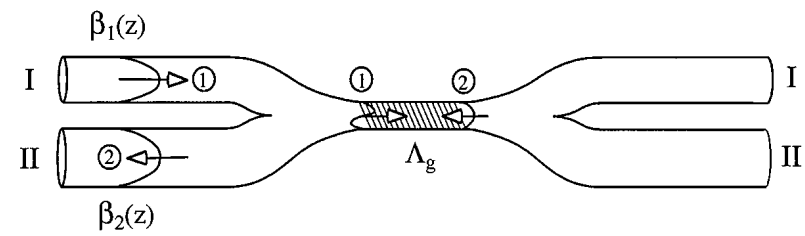

(b)

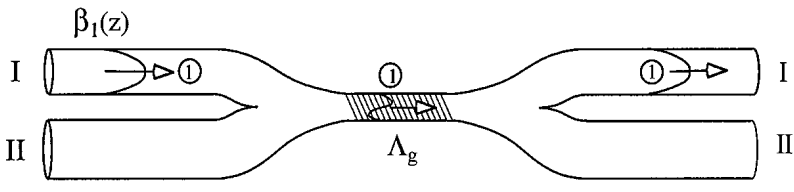

(c)

Fig. 1. Operating principle of the grating-assisted mode converter-coupler: (a) adiabatic mode evolution without a grating, (b) mode transformation when $\beta_{1}+\beta_{2}=2 \pi / \Lambda_{g}$, (c) mode transformation when $\beta_{1}+\beta_{2} \neq 2 \pi / \Lambda_{g}$. 


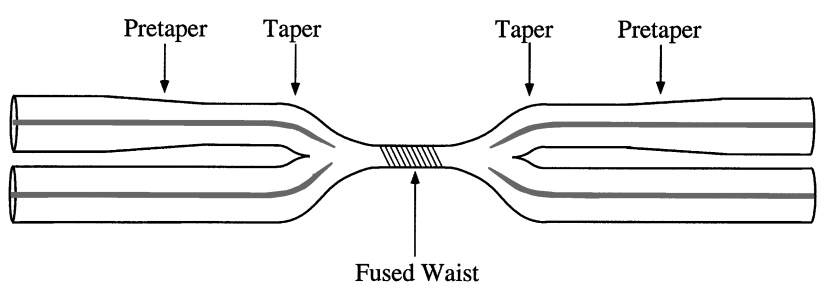

Fig. 2. The structure of the add-drop filter that was fabricated.

fibers before fusion. To record a strong grating in the waist of the device, we used photosensitive single-mode optical fiber. Efficiently coupling the eigenmodes in the merged region and preventing backreflection into the input fiber at a different wavelength $\lambda^{\prime}$ given by $\beta_{1}\left(\lambda^{\prime}\right)=\pi / \Lambda_{g}$ required a transversely asymmetric grating. To satisfy this requirement we recorded a tilted Bragg grating in the waist of the device, using an excimer laser. The tilt angle was $4^{\circ}$.

The out-of-band insertion loss of the filter measured in throughput was $\sim 0.1 \mathrm{~dB}$, including the losses that were due to splicing, nonadiabaticity of the tapered and merged regions, and the UV-induced background loss. The splicing losses were negligible. Figure 3 shows that $<0.1-\mathrm{dB}$ excess loss was incurred during the fabrication of the device before the UV exposure. The UV-induced background loss was $\sim 0.1 \mathrm{~dB}$. We can reduce this loss by exchanging the $\mathrm{OH}^{-}$ions in the photosensitive optical fiber with $\mathrm{OD}^{-}$ions. With $\mathrm{OD}^{-}$-exchanged fiber, a zero-insertion-loss add-drop filter is expected.

A narrow-bandwidth $(\sim 0.7-\mathrm{nm})$ grating was recorded in the waist of the device. The spectral response of the filter is shown in Fig. 4. A peak add-drop efficiency of $\sim 98 \%$ centered at $1547 \mathrm{~nm}$ was measured. We used an unpolarized erbium-doped fiber amplified stimulated emission source and an HP 71450B optical spectrum analyzer to obtain the data. The response of the filter was also examined with polarized input light, but no polarization dependence was observed.

A 98\% add-drop filter efficiency is generally not sufficient to provide adequate channel rejection for WDM applications. To get $-30 \mathrm{~dB}$ of rejection, for example, requires a grating efficiency of $99.9 \%$. Because the reflectivity of a standard fiber Bragg grating can easily surpass this value, ${ }^{11}$ much greater add-drop efficiency is expected with further development. With improved channel rejection the device can simultaneously function as both an add and a drop filter. Thus bidirectional operation is possible. One can also make a wavelength-selective optical switch or router by combining a high-efficiency four-port add-drop filter with a standard optical switch, as shown in Fig. 5. Depending on the electrical input to this device, a particular WDM channel can be routed to either one of two output fibers without disturbing the other wavelength channels.

In summary, we have developed and fabricated an all-fiber four-port add-drop filter by recording a Bragg grating in the merged region of two adia- batically tapered and fused optical fibers. A device with low insertion loss, large add-drop efficiency, no polarization dependence, and a narrow spectral bandwidth was demonstrated. As the operation of the filter does not depend on optical interference or directional coupling, strict optical path length control is not required. With increased add-drop efficiency, the filter is expected to apply to numerous dense WDM applications, including wavelength-selective routing and optical switching.

This research was supported, in part, by the U.S. Army Missile Command under contracts DAAH01-96C-R152 and DAAH01-97-C-R043 with funding from the Defense Advanced Research Projects Agency and by the

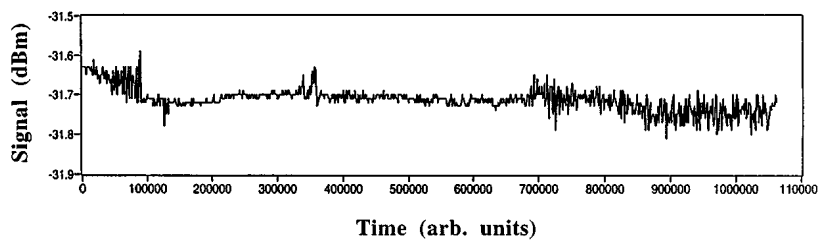

Fig. 3. Throughput of the device during fabrication. Initially the two fibers were separate. After tapering and fusion, but before the UV exposure, $<0.1-\mathrm{dB}$ excess loss was incurred.

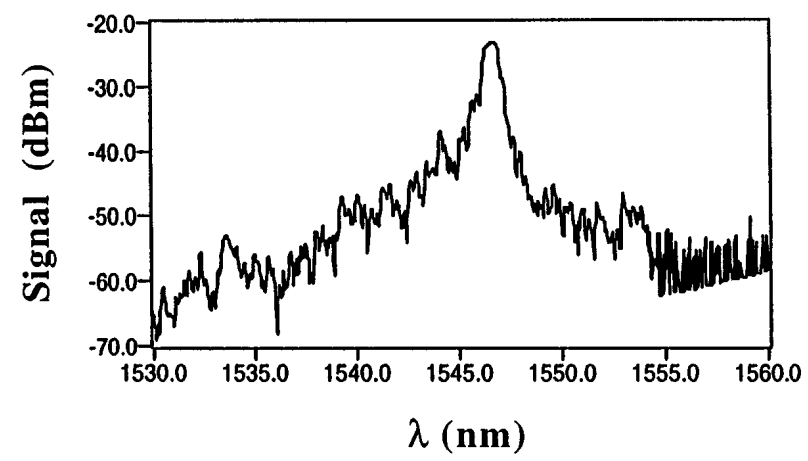

Fig. 4. Spectral response of the add-drop filter.

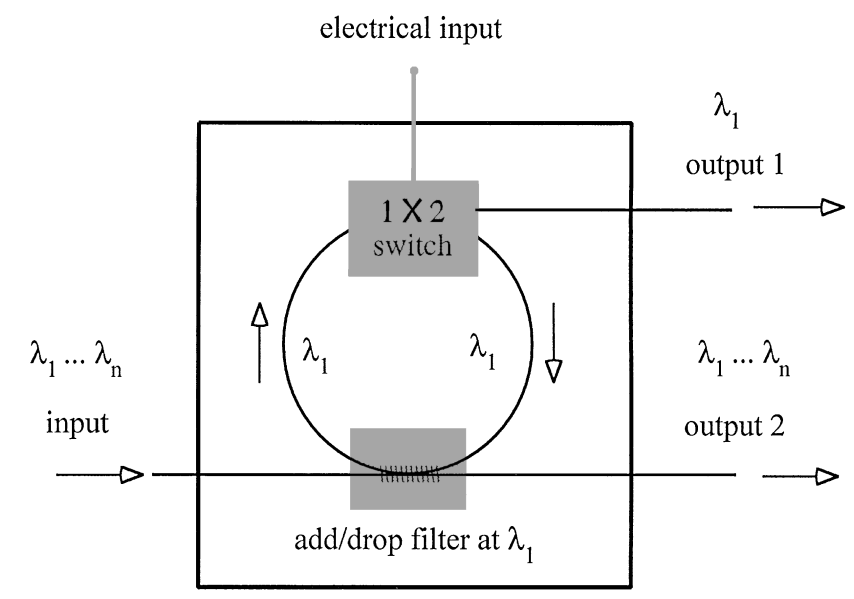

Fig. 5. Wavelength-selective optical switch. 
Naval Surface Warfare Center under contract N0017896-C-3058 with funding from the Ballistic Missile Defense Organization.

*Permanent address, California Institute of Technology, Pasadena, California 91125.

\section{References}

1. C. Dragone, IEEE Photon. Technol. Lett. 3, 812 (1991).

2. F. Heismann, L. L. Buhl, B. I. Miller, M. Newkirk, U. Koren, M. G. Young, and R. C. Alferness, Appl. Phys. Lett. 64, 2335 (1994).

3. T. Komukai, Y. Miyajima, and M. Nakazawa, Jpn. J. Appl. Phys. 34, L230 (1995).

4. J.-L. Archambault, P. St. J. Russell, S. Barcelos, P. Hua, and L. Reekie, Opt. Lett. 19, 180 (1994).
5. I. Baumann, J. Seifert, W. Nowak, and M. Sauer, IEEE Photon. Technol. Lett. 8, 1331 (1996).

6. F. Bilodeau, D. C. Johnson, S. Theriault, B. Malo, J. Albert, and K. O. Hill, IEEE Photon. Technol. Lett. 7, 388 (1995)

7. M. J. Guy, S. V. Chernikov, J. R. Taylor, and R. Kashyap, Electron. Lett. 30, 1512 (1994).

8. D. Marcuse, Theory of Dielectric Optical Waveguides (Academic, Boston, Mass., 1991), Chap. 7.

9. E. Snitzer, "Add-drop device for a wavelength division multiple, fiber optic transmission system," U.S. patent 5,457,758 (October 10, 1995).

10. L. I. Schiff, Quantum Mechanics (McGraw-Hill, New York, 1968), pp. 213-216.

11. R. Kashyap, P. F. McKee, D. J. Armes, M. Shabeer, and D. Cotter, Electron. Lett. 31, 1282 (1995). 